\title{
Quantitative effects of speckle reduction on cross sectional echocardiographic images
}

\author{
R J MASSAY, * R B LOGAN-SINCLAIR, J C BAMBER, $†$ D G GIBSON * \\ From the ${ }^{\star}$ Cardiac and $\ddagger$ Bioengineering Departments, Brompton Hospital, London; and $\dagger$ Institute of Cancer \\ Research, Sutton, Surrey
}

SUMMARY Speckle is prominent on all cross sectional echocardiograms. In order to assess its effects on image quantification, frames from a sector scanner with a six bit grey scale were stored and processed off line to identify and smooth the speckle by means of an adaptive filter based on fully developed speckle. In 14 controls, 12 patients with hypertrophic cardiomyopathy, and 12 with secondary left ventricular hypertrophy, filtering significantly reduced the standard deviation of echo intensity, which was used as a measure of the scatter of pixel amplitude, in all three groups (by $52 \%, 46 \%$, and $46 \%$ respectively). The mean value of back-scattered echo intensity itself, however, was reduced by only $7 \%, 5 \%$, and $8 \%$ respectively, and median values were not affected at all. Mean (SD) left ventricular cavity areas on the apical four chamber view were significantly increased from $26(15)$ to $30(17) \mathrm{cm}^{2}$. The valve dimensions in the parasternal minor axis in 10 patients with mitral stenosis were significantly increased by $11 \%$ laterally, but were unaffected anteroposteriorly. Subjective image quality was appreciably modified: endocardial boundaries in apical views were enhanced and the septal "ground glass" appearance was lost in hypertrophic cardiomyopathy.

Speckle reduction therefore greatly reduced the scatter of pixel values, with little effect on the mean regional back scattered echo amplitude. It also modified the perceived image texture. Improved boundary definition consistently increased the area estimates, particularly when these depended on lateral rather than range resolution.

Speckle is present on all echocardiograms and gives them their characteristic granular appearance. Like noise it degrades the image, but unlike noise it is at least in part determinate-that is it is not random in its origin. Also, unlike electrical noise the global statistical properties of speckle, such as the grey scale histogram and spatial frequency spectrum, are predictable and specific to the imaging system being used. ${ }^{1}$ These differences suggest it might be possible to identify and remove speckle from images by processing them.

We investigated such a system, based on adaptive filtering, and examined how it affected measurements often made on cross sectional echocardiograms: that is the local echo amplitude, the area enclosed by the ventricular cavity, and the dimensions of mitral valve orifice.

Requests for reprints to Dr D G Gibson, Brompton Hospital, Fulham Road, London SW3 6HP.

Accepted for publication 2 May 1989

\section{Patients and methods}

Echocardiograms were recorded with an ATL 860 (6 bit) series scanner that was modified to increase its displayed dynamic range to $45 \mathrm{~dB}$. On the final display, one grey scale level corresponded to $0.86 \mathrm{~dB}$. Images were simultaneously displayed in grey scale and in colour by a Brompton encoder as they were recorded. ${ }^{2}$ Gain settings were standardised: the master gain was increased until the pericardium posterior to the left ventricle was just displayed at the highest level on the encoder. ${ }^{2}$ The depth compensation was then set to a linear ramp across the image at a rate of approximately $2 \mathrm{~dB} / \mathrm{cm}$. Gain settings were not altered thereafter. Standard echocardiographic cuts were used and images were stored on U-matic 3/4 inch video tape by a Sony VO 5800PS recorder.

Figure 1 shows the principle of the speckle reduction system we used. ${ }^{1}$ It is based on the observation that an echocardiogram may show texture even when there is no resolvable or partially resolvable structure in the region being studied. Image texture of this sort 


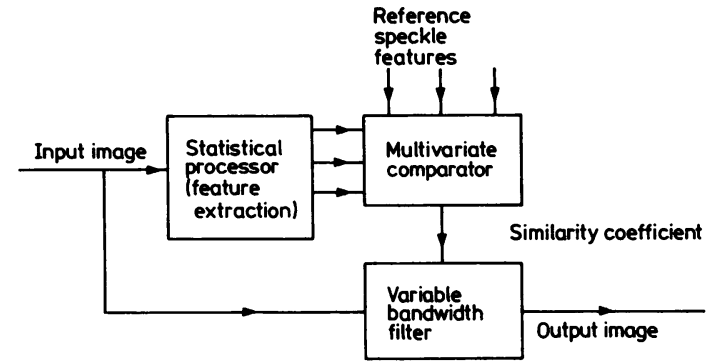

Fig 1 Principle of the adaptive filter used to reduce speckle.

is referred to as fully developed speckle; its characteristics depend primarily on the imaging system used rather than on the target. Structure, resolvable or partially resolvable, only becomes apparent on the image when it modifies the background pattern of fully developed speckle. The system we used examined each small region of the image to determine how much its pattern of local pixel intensity resembled that of fully developed speckle. An index of similarity was thus derived, which controlled the spatial bandwidth of a smoothing filter. Regions of the image that most closely resemble fully developed speckle were heavily smoothed-that is replaced by the local mean -while regions that were least similar were not smoothed at all, leaving the original echo texture intact. We classified texture in terms of a variable, $p$, which represents the ratio of the local mean square pixel level to the local mean. The filtering process consists of modifying the original pixel intensity $(x)$ to a new value $(\hat{\mathbf{x}})$, given by:

$$
\hat{\mathbf{x}}=\overline{\mathbf{x}}+\mathbf{k}(\mathbf{x}-\overline{\mathbf{x}}),
$$

where $x$ represents the local mean, and $k$ is given by the relation:

$$
\mathbf{k}=\mathbf{g p}-\overline{\mathrm{p}} \mathbf{s}) / \mathrm{p},
$$

where $\bar{p} s$ is the mean value of $p$ in a region of fully developed speckle, and $g$ is a scaling factor that determines the overall strength of the smoothing. The value of $k$ has to be $\leqslant 1$, because if it is higher the edges become enhanced. Thus we computed the value for $\mathbf{g}$ for every tenth pixel matrix that gave a value of $k$ just $<1$ over the whole image. Our algorithm differs slightly from that described by Bamber and Daft, ${ }^{1}$ who used the ratio of local variance to local mean as the index of similarity, but found that it performed more satisfactorily for cardiological work where the data had undergone logarithmic compression and where the image contained large and small echo amplitudes close together.

The system was implemented on a Tandon PCA desk top computer. We derived the pixel distribution for fully developed speckle for our echocardiograph from a standard Nuclear Associates tissue-equivalent phantom at mid-depth. All scatterers within it, except for a set of wires, were assumed to be very small in comparison with the wavelength of ultrasound used. A matrix size of $5 \times 5$ pixels proved most satisfactory for processing, and values for $g$ were usually in the range 1.3-1.5 depending on the image being studied. Video frames to be analysed were transferred from tape to a $512 \times 256 \times 6$ bit frame store. We identified appropriate areas of interest, approximately $15 \times 15$ pixels; the local mean, median, and standard deviation were calculated for each and printed out, along with numbers of pixels at each of the 52 grey scale levels used. The frequency histogram was also displayed in analogue form on the image; this proved useful when we studied myocardial echoes because an obviously bimodal form usually meant that we had mistakenly included specular echoes (that is those reflected from large flat surfaces) within the area of interest. We could filter either the area of interest alone or the whole image; the whole image took approximately $70 \mathrm{~s}$ to complete when the matrix size was $5 \times 5$ pixels.

\section{EFFECT OF SPECKLE REDUCTION ON A PHANTOM}

Figure 2 shows the effect of speckle reduction on an image arising from a standard phantom. The unprocessed image (top panel) shows high amplitude echoes arising from wires embedded in it against a background of speckle. The middle panel shows the effect of the filter. The images of the wires are unaltered-indeed, their boundaries seem even more sharply defined than on the unprocessed image, but the speckle is much less prominent. The lowest panel is the result of subtracting the upper panel from the middle panel. Its amplitude has been doubled for clarity. It thus shows what has been removed from the original image by the filter. There is clearly no structural information at all here. Instead its texture is granular and virtually uniform. The unprocessed image can thus be thought of as combining this speckle pattern with the structural information shown in isolation in the middle panel.

\section{EFFECT OF SPECKLE REDUCTION ON BACK - SCATTERED AMPLITUDE IN PATIENTS AND CONTROLS}

The effect of reducing speckle on the amplitude of back-scattered echoes from myocardium was studied in 12 patients with typical hypertrophic cardiomyopathy (diagnosed on clinical and echocardiographic criteria), 12 with left ventricular hypertrophy secondary to valvar aortic stenosis severe enough to need valve replacement, and 14 normal controls. Areas of interest were located in the basal and midportions of the septum and posterior wall on the parasternal long axis cut, ${ }^{3}$ excluding high amplitude 


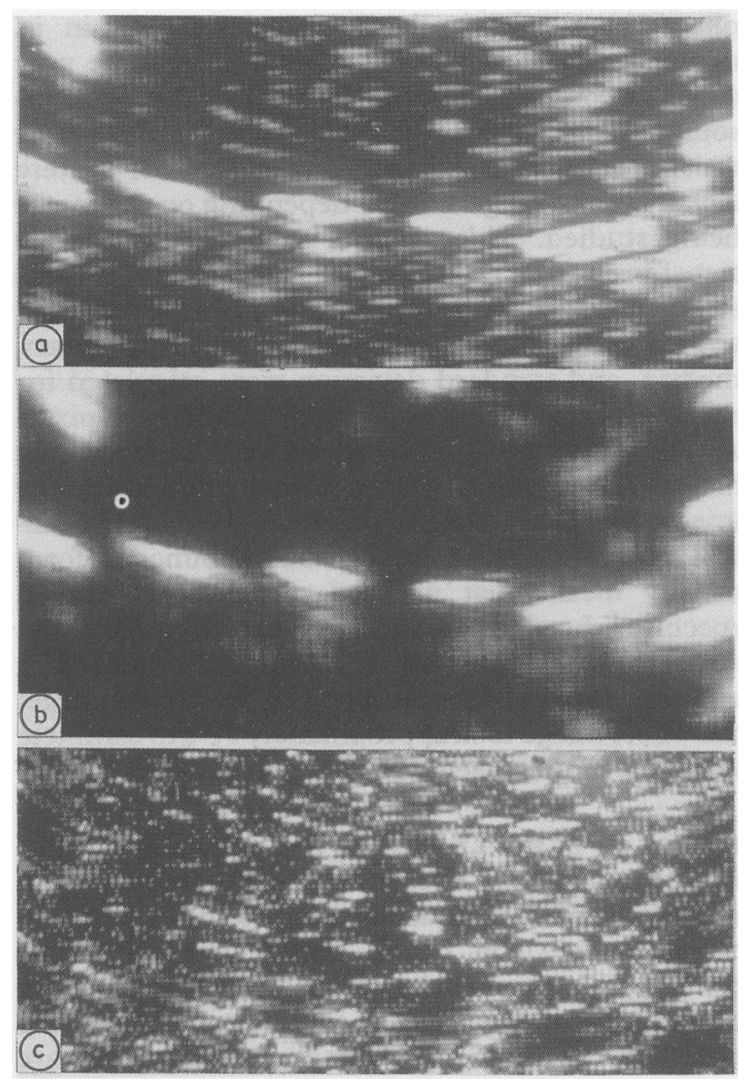

Fig 2 Effect of speckle reduction on the echograms of a standard phantom. The upper panel is the original image, with high amplitude signals arising from wires embedded within the phantom. The middle panel shows the same video frame after speckle reduction. Note that the amplitude of the background speckle is greatly reduced, while the images of the wires are virtually unaffected. The bottom panel, derived by subtracting the middle from the upper panel, shows what has been removed by the filter. The overall amplitude in this frame has been doubled for clarity.

echoes arising from endocardium or pericardium. We derived the mean pixel intensity and its standard deviation for each of these areas before and after applying the speckle reduction algorithm to the area of interest.
MEASUREMENT OF MITRAL VALVE DIMENSIONS AND LEFT VENTRICULAR CAVITY AREAS

We assessed how speckle reduction affected estimates of mitral valve dimensions from parasternal short axis cuts at the level of the valve cusps in 12 patients with rheumatic mitral stenosis. We photographed single frames on $35 \mathrm{~mm}$ film, along with the depth calibration before and after processing. With the image magnified 3-4 times, we measured the apparent dimensions of the valve orifice in the anteroposterior direction by the leading edge method and in the lateral direction from the "inside" of the cusp echoes.

We measured left ventricular cavity area on echocardiograms recorded in the apical four chamber cut in 11 patients with various cardiac conditions, including aortic regurgitation (two), rheumatic valve disease (three), cardiomyopathy (two), and four controls. Again we photographed the images on $35 \mathrm{~mm}$ film before and after processing and projected them on to a digitising table, outlining the area of the cavity as we have previously described for contrast angiograms. ${ }^{5}$ On both processed and unprocessed images, we took the inner border of the echoes forming the cavity outline to be the position of the endocardium.

\section{Results}

\section{BACK-SCATTERED AMPLITUDE}

The table shows the values for the mean and standard deviation of back-scattered amplitude for controls and patients with primary and secondary left ventricular hypertrophy. Values from controls tended to be lower than those from patients with ventricular hypertrophy, but did not differ significantly. Similarly, mean values did not differ between patients with primary and secondary hypertrophy or between regions. Speckle reduction caused a very consistent reduction $(46 \%-52 \%)$ in the standard deviations of pixel intensity in all three groups and values fell in every individual. The effect on the mean level of pixel intensity was very much smaller, ranging from a fall of $4.8 \%$ to a fall of $7 \cdot 0 \%$. These values were significantly less than the change in standard deviation ( $p<0.001$ for all three groups).

Table Effect of filtration on myocardial echo intensity

\begin{tabular}{|c|c|c|c|c|c|c|}
\hline \multirow[b]{2}{*}{ Group } & \multicolumn{3}{|c|}{ Unfiltered values } & \multicolumn{3}{|c|}{ Change with filtration (\%) } \\
\hline & Mean & $S D$ & Median & Mean & $S D$ & Median \\
\hline $\begin{array}{l}\text { Control } \\
\text { Hypertrophic cardiomyopathy } \\
\text { Secondary left ventricular hypertrophy }\end{array}$ & $\begin{array}{l}7 \cdot 3 \\
8 \cdot 2 \\
9 \cdot 0\end{array}$ & $\begin{array}{l}4 \cdot 4 \\
4 \cdot 2 \\
4 \cdot 4\end{array}$ & $\begin{array}{l}6 \cdot 3 \\
7 \cdot 7 \\
7 \cdot 7\end{array}$ & $\begin{array}{l}-7 \cdot 0 \\
-5 \cdot 9 \\
-4 \cdot 8\end{array}$ & $\begin{array}{l}-52 \\
-46 \\
-46\end{array}$ & $\begin{array}{l}-0 \cdot 7 \\
-2 \cdot 3 \\
-1 \cdot 5\end{array}$ \\
\hline
\end{tabular}

All the differences between the percentage change in standard deviation for median or mean values were significant $(p<0.001)$. 

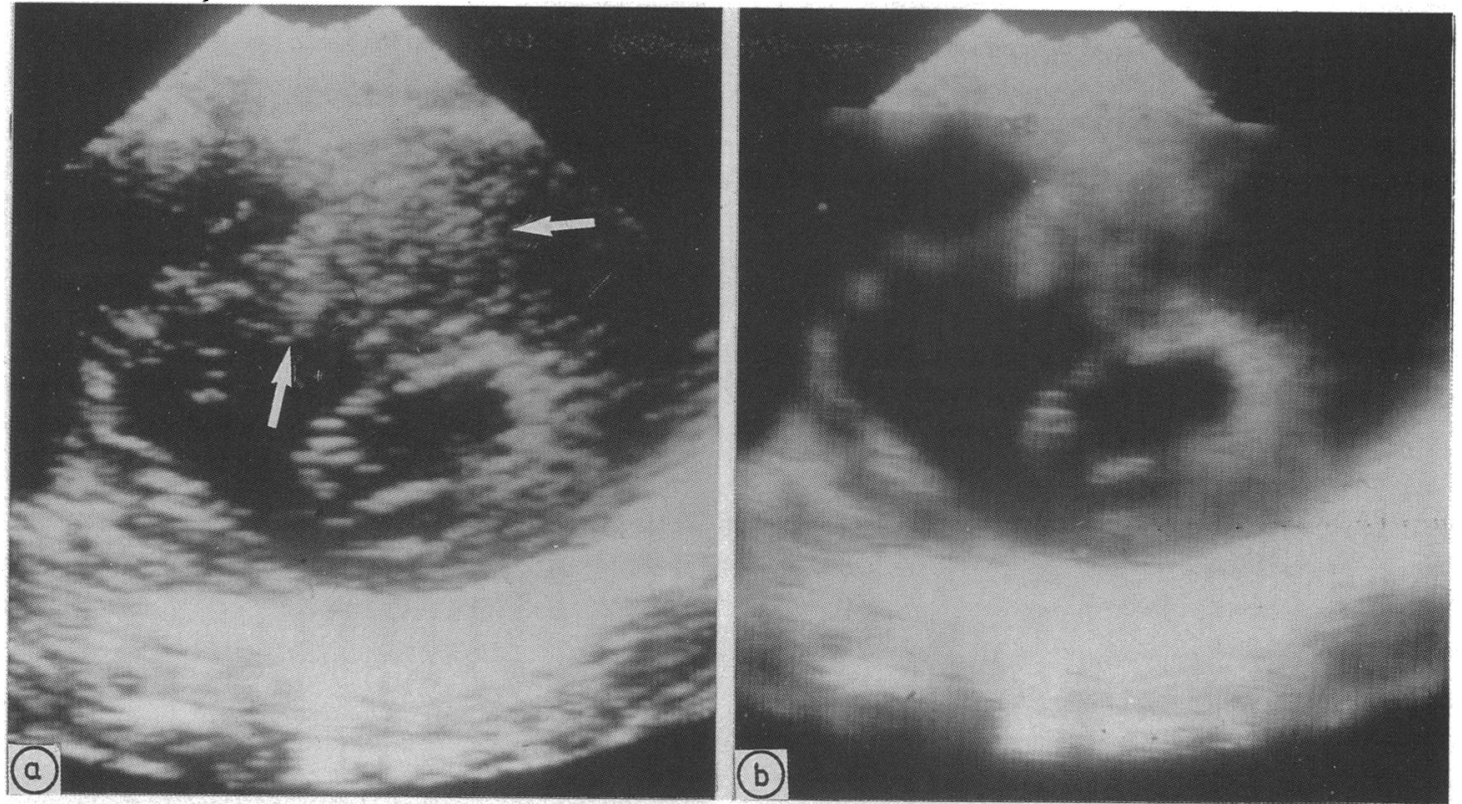

Fig 3 Effect of speckle reduction on myocardial echo texture in a patient with hypertrophic cardiomyopathy (parasternal minor axis cut). Note that the gramular appearance of the myocardium in the original image (left, arrows) has been removed by processing, and replaced by a more diffuse structure.

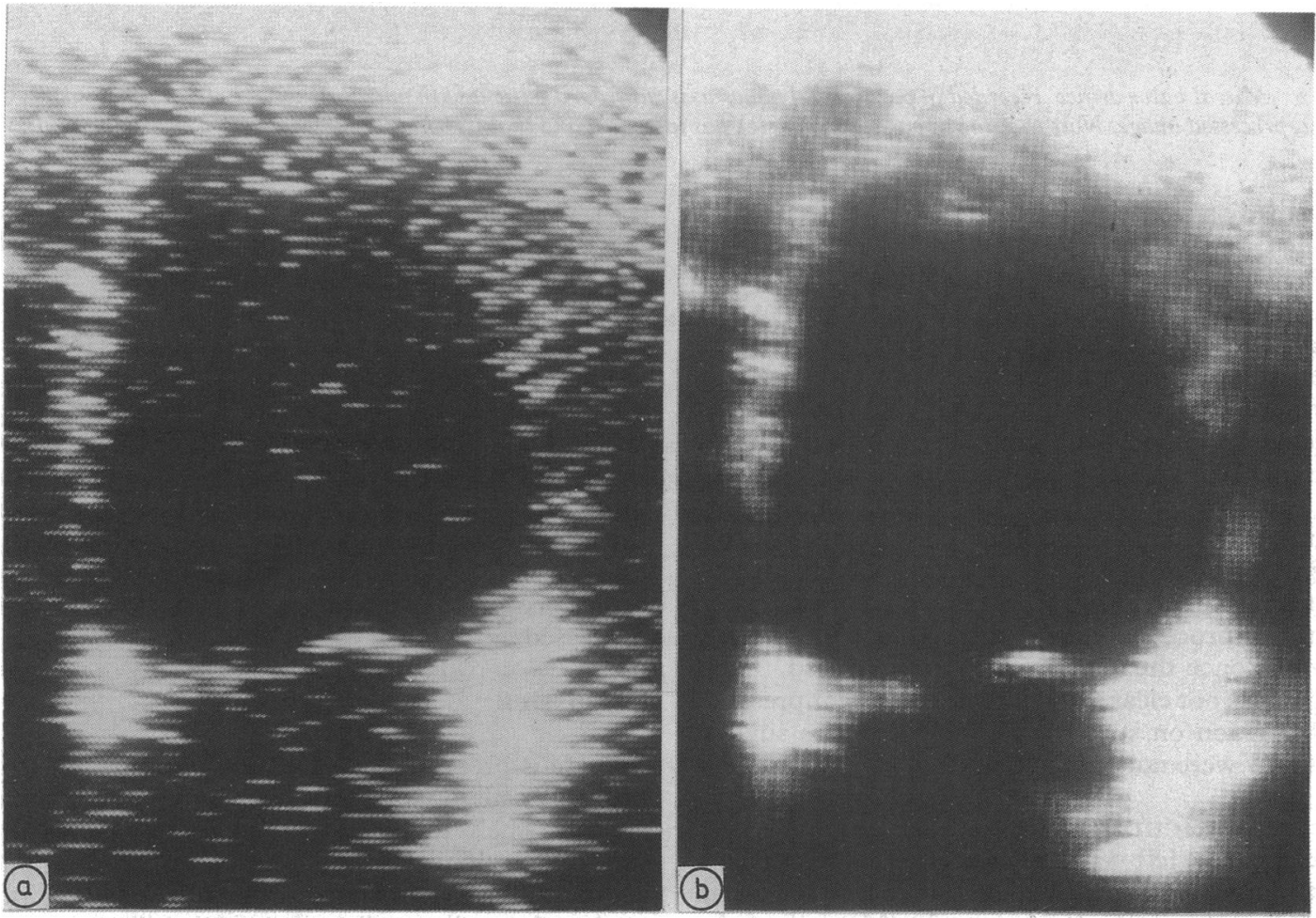

Fig 4 Left ventricular echocardiogram (apical four chamber cut) showing the effect of speckle reduction on the apparent cavity size and endocardial boundary. Left, original image; right, filtered image. 


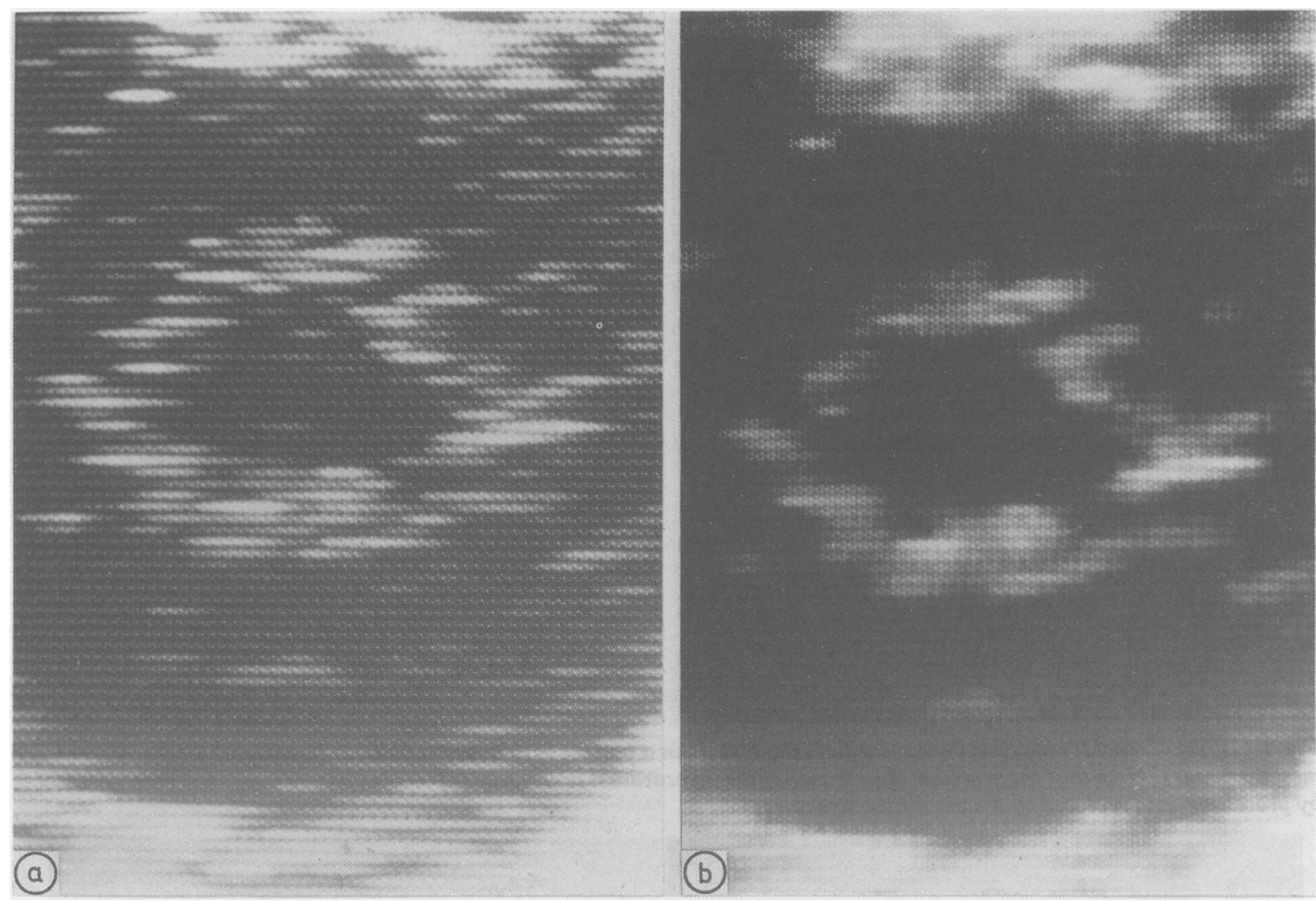

Fig 5 Mitral valve orifice, recorded in parasternal minor axis cut from a patient with mitral stenosis. Left, original image; right, processed image. Note that the apparent orifice size has increased and that its boundary appears more clearly defined.

The median pixel level did not show any consistent change.

Reducing speckle caused the images to change strikingly. In the patients with left ventricular hypertrophy, whether primary or secondary, echo texture often seemed abnormal before processing, with an obvious granular appearance (fig 3, left panel). After speckle reduction, this granularity was no longer present (right panel) and was consistently replaced by a diffuse pattern of echoes within the myocardium. Apparently randomly distributed, sharply defined sonolucent areas were strikingly accentuated; although in retrospect they had been present on the unprocessed image, they became much more obvious once the speckle had been reduced. Their origin was not clear though their consistent presence and position on successive frames seem to suggest that they were not artefactual.

\section{LEFT VENTRICULAR CAVITY AREA}

The apparent left ventricular cavity area was consistently larger after speckle reduction (fig 4). The mean value rose from $26(15) \mathrm{cm}^{2}$ to $30(17) \mathrm{cm}^{2}$ on the processed image $(p<0.002)$, the increase being at least $1 \mathrm{~cm}^{2}$ in each patient. Subjectively, we noted that the internal boundary of the cavity became much clearer, making it easier to digitise (fig 4).

\section{ORIFICE DIMENSIONS OF THE MITRAL VALVE}

On the parasternal minor axis view, mitral valve dimensions in the anteroposterior direction were unaffected by speckle reduction, the mean (SD) being unchanged at $21(12) \mathrm{mm}$. The transverse dimension, however, increased from 36 (9) $\mathrm{mm}$ on the unprocessed image to (40) (12) after filtering (fig 5) ( $<<0.002)$. Again, the apparent boundary of the orifice became much clearer after the image had been processed.

\section{Discussion}

Speckle is prominent in all ultrasound images. It is probably caused when wave fronts from targets that are too small to be individually resolved at the wavelength of ultrasound used interfere with one another. The exact pattern seems to depend mainly on the echocardiograph used, and it is still not certain whether it conveys any structural information at all. 
The overall result of speckle, however, is to degrade fine detail, so it was of interest to examine how cardiological images were altered when it was substantially reduced.

There are several ways in which this might have been done. Speckle can be treated simply as noise and reduced by frame averaging or smoothing. While this would certainly reduce its overall effect, spatial and temporal information would also be lost. A second approach would be to use more than one transducer -again this might have been possible with a static organ, but it would be quite impracticable for the heart without losing diagnostically valuable information. Our approach identified the speckle pattern characteristic of the echocardiograph we used, determined its statistical features, and smoothed it on the image. This approach proved feasible, and it may well be possible to implement it in real time. The median pixel level did not change, and the mean pixel level fell very slightly. The scatter of pixel levels around these mean values, however, was strikingly reduced. Using the phantom, we showed that our ability to detect high frequency detail was unaffected. Further, had there been any non-specific smoothing, we would have found it on the subtraction image where it would have appeared as high frequency boundaries to the structural features. Altogether, the way regional amplitude and local pixel distribution changed was quite compatible with speckle reduction, and cannot be explained by simple smoothing of the image.

It seems to be generally accepted that echocardiograms are granular, and thus qualitatively different from the images produced by other methods such as computed tomography or magnetic resonance imaging. Does a granular texture reflect anything fundamental about myocardium? There is some clinical evidence to suggest that it might. A "ground glass appearance" of the septum is said to be characteristic and even diagnostic of hypertrophic cardiomyopathy. ${ }^{4}$ Myocardial texture has been analysed on the basis of the presence, distribution, and size of what are described as "highly refractile bodies". 6 The myocardium is unusual among the cardiac structures commonly studied by echocardiography, in being detected almost entirely from back-scattered echoes rather than from specular reflection, as are the valves and endocardial surfaces. These back-scattered echoes arise from targets that are small compared with the wavelength of ultrasound used, and so the resulting image is likely to be more sensitive to the effects of speckle than one based mainly on specular echoes. Not surprisingly, therefore, the effect of reducing speckle by a system based on single feature criteria on myocardial texture was profound. It no longer appeared granular, even in patients with hypertrophic cardiomyopathy or secondary left ventricular hypertrophy, implying that the apparent texture in the unprocessed images had corresponded very closely with the criteria we used to identify fully developed speckle. By contrast, median pixel levels, which are not affected by speckle, were unaltered. We conclude that the granular appearance within the myocardium in these patients need not reflect underlying structure in any significant way. We did not establish whether the appearances shown by filtering had any anatomical basis. This will require further study.

Areas are often measured routinely on cross sectional images so we studied two examples. In patients with rheumatic mitral stenosis the anterioposterior dimension of the valve orifice was unaffected by filtering the image but the lateral dimension was consistently larger, so that the calculated area increased. The apparent left ventricular cavity area as assessed in the apical four chamber view was approximately $15 \%$ larger when measured on processed images. This increased to $25 \%$ when the volume was estimated. These differences are large enough to be clinically significant. The effect of processing on the apparent area seemed to be related to the way in which the image of the corresponding boundary was generated. On the short axis parasternal cut that is traditionally used to measure mitral valve area a leading edge defines the apparent orifice only along the anterior surface of the posterior cusp. The trailing edge of the anterior cusp forms its anterior border, while the commissures are defined by lateral resolution. Thus both they and the ventricular cavity on the apical four chamber view are outlined by backscattered rather than reflected echoes and so they are likely to be particularly sensitive to the effects of superimposed speckle. It follows that even a measurement so seemingly unambiguous as the area enclosed by what appear to be clear anatomical boundaries on an echocardiogram depends critically on how the image is generated.

Smoothing, ${ }^{7}$ frame averaging, ${ }^{8}$ boundary enhancement, ${ }^{10}$ and colour coding ${ }^{2}$ have been examined for their possible value in processing echocardiograms. All these manoeuvres have in common a non-specific algorithm, so their value is limited to accentuating information already present on the unprocessed image. More complex analysis requires that the processes underlying image generation be better understood, so that confounding factors can be identified and removed. Speckle reduction seems to be a step in this direction, though the physics of its production do not have to be understood in detail for its effects to be quantified and isolated. We hope to improve these methods so that they can be used in real time, rather than simply 
on individual frames. Ideally, echocardiographs would be designed to eliminate speckle as the original data are collected rather than to remove it retrospectively from the final image as we have done. As the interaction between ultrasound and tissues becomes more clearly understood, we expect that equipment design will improve so that the resolution and information content of images will correspondingly increase.

\section{References}

1 Bamber JC, Daft C. Adaptive filtering for reduction of speckle in ultrasonic pulse-echo images. Ultrasonics 1986;5:41-4.

2 Logan-Sinclair R, Wong CM, Gibson DG. Clinical application of amplitude processing of echocardiographic images. Br Heart $J$ 1981;45:621-7.

3 Edwards WD, Tajik AJ, Seward JB. Standardized nomenclature and anatomic basis for regional tomographic analysis of the heart. Mayo Clin Proc 1981; 56:479-97.

4 Martin RP, Rakowski H, French J, Popp RL. Idiopathic hypertrophic subaortic stenosis viewed by wide-angle phased array echocardiography. Circulation 1979;59:1206-17.

5 Gibson DG, Prewitt TA, Brown DJ. Analysis of left ventricular wall movement during isovolumic relaxation and its relation to coronary artery disease. Br Heart J 1976;38:1010-9.

6 Bhandari AK, Nanda NC. Myocardial texture characterization by two-dimensional echocardiography. $\mathrm{Am}$ J Cardiol 1983;51:817-25.

7 Parker DL, Pryor TA, Ridges JD. Enhancement of two-dimensional echocardiographic images by lateral filtering. Comp Biomed Res 1979;12:265-77.

8 van Ocken E, Claes V, Brutsaert DL. Image enhancement by digital averaging of 2-dimensional echopictures. In: Latin V, ed. Proceedings: Fourth European Congress on Ultrasound in Medicine. Amsterdam: Excerpta Medica, 1981:69.

9 Grube E, Becher H, Backs B. Automatic contour finding and digital subtraction technique in 2-dimensional echocardiography. In: Roelandt J, ed. Digital techniques in echocardiography. Dordrecht: Martinus Nijhoff, 1987:99-122.

10 Skorton DJ, McNary CA, Child JS, Newton FC, Shah PM. Digital image processing of two dimensional echocardiograms; identification of the endocardium. Am J Cardiol 1981;48:479-86. 